\title{
Is it bronchiolitis obliterans syndrome or is it chronic rejection: a reappraisal?
}

\author{
G.M. Verleden*, L.J. Dupont* and D.E. Van Raemdonck*
}

ABSTRACT: Chronic rejection (obliterative bronchiolitis) is the single most important cause of chronic allograft dysfunction and late mortality after lung transplantation. As this condition is difficult to prove using biopsy specimens, a clinical term, bronchiolitis obliterans syndrome (BOS) has been in use for $>10$ yrs to describe the progressive decrease of pulmonary function. However, before diagnosing a patient as having BOS, based on a sustained and progressive decrease in forced expiratory volume in one second and/or forced mid-expiratory flow between 25-75\% of forced vital capacity, different confounding factors have to be eliminated.

Treatment of BOS mainly consists of an increase or a change in the immunosuppressive drug regimen, which may lead to more pronounced infectious complications. Recently, two new options have become available to treat patients with BOS, treatment of gastro-oesophageal reflux and azithromycin.

In the present paper, the authors give an overview of the current data on these two modalities, which may lead to a restoration of the pulmonary function in some of the patients, illustrating once more the fact that bronchitis obliterans syndrome is not always a manifestation of chronic rejection.

\section{KEYWORDS: Bronchiolitis obliterans syndrome, chronic rejection, lung transplantation}

hronic allograft dysfunction, due to chronic rejection with obliterative bronchiolitis (OB) as the pathological hallmark, remains the leading cause of mortality after lung transplantation [1]. The overall prevalence of $\mathrm{OB}$ in patients surviving $>3$ months after transplantation is estimated at $64 \%$ after heart-lung transplantation and $68 \%$ after lung transplantation [2]. The actuarial 1, 3 and 5-yr freedom from $\mathrm{OB}$ is 82,42 and $25 \%$, respectively, in adults [3], and 76 and $37 \%$ after 1 and 3 yrs in children [4]. In the Groningen experience and in Leuven (in adults only), the actuarial freedom from BOS was $55 \%$ and $40 \%$, respectively, after $5 \mathrm{yrs}[5,6]$. The incidence is indeed estimated at $12 \%$ at $1 \mathrm{yr}$ and $>50 \%$ at $5 \mathrm{yrs}$ [7]. Chronic rejection after lung transplantation is the single most important factor responsible for late mortality, with as many as $40-50 \%$ of deaths $>3-$ 5 yrs after transplantation being due to chronic rejection [1].

\section{CLINICAL CHARACTERISTICS}

OB was first described in 1984 in a patient who developed a progressive decline in forced expiratory volume in one second FEV1 after a heart and lung transplantation [8]. Chronic allograft rejection is consistently characterised by a reduction in pulmonary function parameters, most specifically in FEV1, attributed to airways obstruction. There is no significant reversibility after inhalation of short-acting $\beta_{2}$-agonists. The onset of symptoms is mostly insidious, with progressive exertional dyspnoea, often accompanied by cough. Sometimes an airways infection seems to have triggered the onset of $\mathrm{OB}$, with quite acute dyspnoea, wheezing and the development of airways obstruction. Later in the course of the disease, airways superinfections are frequently seen and colonisation with Pseudomonas aeruginosa and Aspergillus fumigatus is common. At that time, high-resolution computerised axial tomography (CAT) scan of the thorax often reveals bronchiectasis and other signs of chronic infection. Auscultation of the lungs is often normal. However, occasional rhales, and in later stages, squeaks may be heard. Once established, OB may develop progressively and lead to severe airways obstruction with respiratory insufficiency and death resulting from an infectious exacerbation. In other patients, the progression may be arrested, either spontaneously or in response to treatment [9]. Sometimes, episodes of progressive loss of lung function disperse with stable intervals.
AFFILIATIONS

*Depts of Respiratory Diseases and Lung Transplantation Unit, and

\#Dept of Thoracic Surgery,

University Hospital Gasthuisberg 49, Herestraat, B-3000 Leuven, Belgium.

CORRESPONDENCE

G.M. Verleden

University Hospital Gasthuisberg Dept of Respiratory Diseases and Lung Transplantation Unit 49 Herestraat B-3000 Leuven Belgium Fax: 3216346803 E-mail: geert.verleden@uz. kuleuven.ac.be

Received:

May 132004

Accepted after revision:

October 022004 


\section{DIAGNOSIS}

$\mathrm{OB}$ is a pathological term, reflecting a scarring process affecting the small noncartilaginous airways of the lung graft [10]. The patchy nature of OB, particularly in its early stages, means that transbronchial biopsy (TBB) often has a poor yield in this diagnosis. In fact, it has been demonstrated that the sensitivity of TBBs for OB was only $28 \%$, whereas the specificity was $75 \%$ [11]. In another study by KRAMER et al. [12], only $15 \%$ of TBB showed signs of OB, whereas all investigated patients had clinical OB.

As a result, in 1993, a committee sponsored by the International Society for Heart and Lung Transplantation proposed a clinical definition, called bronchiolitis obliterans syndrome (BOS) to describe the progressive loss of pulmonary function after (heart) lung transplantation [13]. Based on pulmonary function criteria, rather than histology, BOS has been initially divided into four, and more recently into five stages [14]. Subtypes a and b exist within each BOS category; these are based on either no pathological evidence of $\mathrm{OB}$ or no pathological material for evaluation (a) or pathological evidence of $\mathrm{OB}(\mathrm{b})$.

\section{DOES BOS EQUAL OB?}

BOS is used to suggest chronic graft dysfunction secondary to persistent airflow obstruction. Airflow obstruction is defined as a persistent (i.e. two consecutive measurements within 3-6 weeks) decline of $\geqslant 20 \%$ (BOS stage 1 ) from baseline FEV1, defined as the mean of the two post-operative best FEV1 values (with $\geqslant 3$ weeks in between the measurements). Since it became apparent from other studies that the forced midexpiratory flow between $25-75 \%$ of forced vital capacity may decline earlier than the FEV1 $[5,15,16]$, this parameter has now been included in the recent revision of the diagnostic criteria for BOS [14].

Although BOS has been proposed as a clinical description of $\mathrm{OB}$, it is obvious that not all patients in whom airflow obstruction develops have BOS and, hence, OB. In fact, several confounding factors have to be excluded before a patient may be diagnosed as having BOS. These factors may originate from the graft itself or from the native lung and include infection, acute rejection, anastomotic problems, disease recurrence, ageing, native lung hyperinflation, disease progression and factors that induce a restrictive defect such as pleural disease, steroid myopathy, pain, etc. [14]. Fiberoptic bronchoscopy with TBBs and less frequently thoracoscopic or open lung biopsies can, therefore, be useful in the clinical setting of BOS in confirming or excluding alternative or concomitant diagnoses.

Several risk factors for the development of BOS have been identified, including alloimmune-dependent mechanisms, such as the number and severity of acute rejection episodes, lymphocytic bronchitis/bronchiolitis, but also nonalloimmunedependent mechanisms, such as viral infections (especially Cytomegalovirus (CMV) infections) and possibly also non$\mathrm{CMV}$ viral and bacterial infections [14]. However, even in the presence of these known risk factors for the development of BOS and after exclusion of all confounding factors, as mentioned above, the following case report may illustrate that BOS does not necessarily reflect chronic rejection, a mostly irreversible process, which has been suggested by ESTENNE et al. [14] in their revised BOS criteria paper.

\section{Case report}

After a heart and lung transplantation for Eisenmenger's syndrome, the patient developed recurrent acute rejections, for which high-dose i.v. steroids were administered; cyclosporin was changed to tacrolimus, and azathioprine to mycophenolate mofetil (fig. 1). Despite these immunosuppressive changes, the patients FEV1 gradually deteriorated, finally ending in BOS stage 2. Repeated TBBs did not reveal any acute rejection, nor were there signs of OB. Even two 14-day courses of rabbit antithymocyte globulin did not improve the patient's pulmonary function. Other confounding factors which might interfere with the diagnosis of BOS were excluded. Due to the recurrent acute rejection episodes, the gradual decline in FEV1 without any other explanation or a therapeutic response to augmented immunosuppressive therapy, the present authors concluded that this patient had BOS due to chronic rejection.

Finally, the patient was treated with Azithromycin, in addition to her current immunosuppressive drug regimen, and within 4 weeks FEV1 had normalised.

This case evolution has recently been corroborated by two observations discussed in further detail below.

\section{GASTRO-OESOPHAGEAL REFLUX AND BOS}

Gastro-oesophageal reflux (GER) is regarded as a hypothetical risk factor for the development of BOS [14] and has been demonstrated to be a reversible cause of allograft dysfunction or BOS after lung transplantation [17]. Moreover, lung transplantation on its own significantly increased the incidence of GER, as measured objectively by $24-\mathrm{h}$ pH studies, despite a lack of symptoms in most patients. In fact, before lung transplantation, $35 \%$ of the patients had an abnormal acid

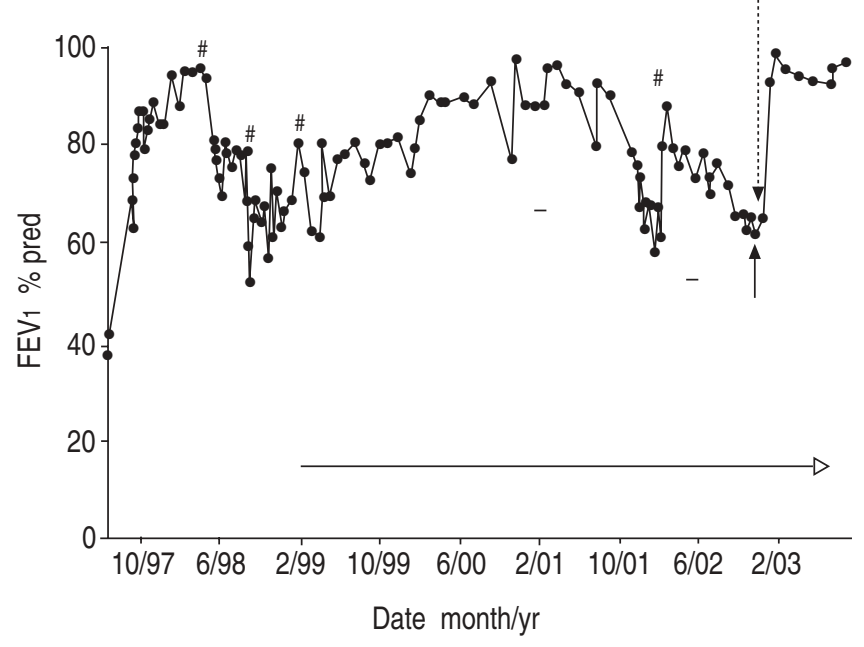

FIGURE 1. Forced expiratory volume in one second (FEV1) evolution after transplantation. Despite a change from cyclosporin and azathioprine to tacrolimus and mycophenolate mofetil (open arrow) and two 14-day courses of rabbit antithymocyte globulin (-), the patient experienced a gradual decline in the FEV from $2.91 \mathrm{~L}(95 \%$ of predicted) to $1.8 \mathrm{~L}(62 \%$ pred), bringing the patient into bronchiolitis obliterans syndrome (BOS) stage 2 (solid arrow). After adding azithromycin (dotted arrow), this restored the patients $\mathrm{FEV}_{1}$ to $2.76 \mathrm{~L}$, which was maintained during the 16 months of treatment up to the last measurement. \#: a biopsy-proven acute rejection episode, which was treated with a 3-day course of i.v. steroids $\left(1 \mathrm{~g} \cdot \mathrm{day}^{-1}\right)$. 
contact time, whereas after transplantation, this increased to $65-69.8 \%[18,19]$. After fundoplication, there was a significant survival difference, together with an improvement of the pulmonary function [20]. Of the 43 patients who underwent fundoplication in the series by DAVIS et al. [20], 26 met the criteria for BOS at the time of the operation (13 patients in stage 1 , seven in stage 2 and six in stage 3 ). After the fundoplication, 16 patients had improved their BOS stage: 10 of 13 BOS stage 1 patients and three of seven BOS stage 2 patients no longer met the criteria for BOS, whereas another two from the BOS stage 2 group improved to BOS stage 1 . Of the six patients in BOS stage 3, only one improved [20].

The mechanism by which lung transplantation increases the incidence of GER is probably multifactorial, including vagus nerve dysfunction and effects of immunosuppressive medication on gastric emptying and lower oesophageal sphincter function. In fact it has previously been demonstrated that immunosuppressive agents may indeed influence gastric emptying, for instance cyclosporin A causes a slower solid emptying pattern, whereas tacrolimus produces a significantly faster solid emptying [21]. That explains why conversion from cyclosporin to tacrolimus may improve gastric discomfort in transplant patients [22].

How GER produces chronic allograft dysfunction (BOS) is not quite clear, although lung denervation with impaired cough reflex and abnormal mucociliairy clearance will certainly have a role to play. These factors might increase the acid contact time and so prolong the injury time to the airways. This may lead to sustained inflammatory reactions with airway hyperresponsiveness as a consequence [23], which is indeed regarded as a risk factor for the development of BOS [24]. Recently, it has also been demonstrated that GER is associated with an increased number of acute rejection episodes and that the presence of GER increased the rapidity and severity of the initial acute rejection. Furthermore, early fundoplication decreased the number of late rejections [25]. These data indicate, as already suggested by ESTENNE et al. [14], that nonalloimmune-dependent mechanisms (in this case GER) may indeed lead to BOS, and may be partly or completely reversible when the causal factor is treated.

\section{AZITHROMYCIN AND BOS}

In a recent pilot study, azithromycin has been shown to produce an amelioration of the FEV1 in lung transplant patients in different stages of BOS. GERHARDT et al. [26] treated six lung transplant patients with BOS with azithromycin and showed a significant improvement of the FEV1 $(+17.1 \%$, or an absolute increase of $0.5 \mathrm{~L}$ ) after a mean follow up of 13.7 weeks [26].

Recently, the current authors also treated eight lung transplant patients with progressive chronic allograft dysfunction (four patients were in BOS stage 1, two in stage 2 and two in stage 3) with azithromycin and demonstrated a comparable improvement of the FEV1 after 12 weeks $(+18.3 \%)$ [27]. The FEV1 started to increase after 2-4 weeks of treatment [27]. In the series by GERHARDT et al. [26], five of the six patients had a significant response, whereas in the recent series [27], five of the eight patients showed significant improvement in their FEV1. However, two nonresponders, in a study by the current authors, experienced at least a temporary stabilisation of their pulmonary function parameters [27]. In the study by VERLEDEN et al. [27], three patients were followed up during 36 weeks and the authors demonstrated that the improvement in their FEV1 persisted during that whole time period. In the case described in the present paper, the treatment has already been administered for 16 months, with a sustained normalisation of the FEV1.

In both series there were patients who no longer met the criteria for BOS, or improved their BOS stage. The study of GERHARDT et al. [26] was the first to show a significant improvement of the FEV1 in BOS patients using medical treatment. Up to now, only a stabilisation of the FEV1 had been achieved in such patients, mostly by an increase or a shift in the immunosuppressive regimen $[9,28]$.

The possible mechanisms of action of azithromycin in lung transplant patients with BOS remain unclear at the present time. Nevertheless, several explanations have been put forward including: 1) inhibition of the transcription of quorum sensing genes, which may prevent production of tissuedamaging proteins and which have indeed been detected in clinically stable lung transplant recipients without any signs of infection [28]; 2) a positive effect on GER, since macrolide antibiotics are known as motilin agonists; and 3) an inhibitory effect on neutrophils and interleukin-8, which are characteristically found in increased amounts in the bronchoalveolar lavage fluid of patients with BOS [29]. However, to date, there are no positive arguments for either mechanism.

\section{CONCLUSIONS}

Although the advice of the revised BOS criteria is to exclude all kinds of confounding factors before interpreting a sustained decline in FEV1 as the result of BOS, and hence chronic rejection, it seems acceptable from recent data that GER can no longer be proposed as a hypothetical but rather as a real confounding factor. Before accepting that a patient with BOS also has chronic rejection or $\mathrm{OB}$, it seems imperative to explore for underlying GER and to consider a fundoplication when positive, although at the present time it is not known how many and specifically which patients will benefit from this procedure. It is unknown whether medical treatment for GER will have comparable results. Therefore, comparative and preferably multicentre trials will have to be performed.

In contrast, azithromycin, which is the first medical treatment to demonstrate a significant and sustained improvement of the FEV1 in patients with BOS, is also worthwhile considering as a treatment option in these patients. The treatment is simple and may prevent the patient from serious side-effects due to an increase of the immunosuppressive treatment, as has been routine procedure up to now in patients with $\operatorname{BOS}[9,28]$.

In the two available studies on this subject, the same starting dose of $250 \mathrm{mg}$ per day for 5 days, followed by $250 \mathrm{mg}$ every other day was used $[26,27]$. However, it is impossible to know whether this is the right dose for every patient and what the ideal length of treatment should be. This is also not hindered by the lack of knowledge on the mechanisms of action of this specific drug. Therefore, these results are sufficiently impressive to encourage a randomised, controlled trial which should be able to answer the dosing question and maybe also the mechanisms question. 
Nonetheless, for the individual patient with an established diagnosis of bronchiolitis obliterans syndrome, it may be interesting to explore the possibility of gastro-oesophageal reflux and to treat it (medically or surgically) or if gastrooesophageal reflux cannot be demonstrated, to give a 4-6 weeks trial of azithromycin before changing or augmenting the immunosuppressive therapy, which give rise to an increased danger of renal dysfunction and infectious complications.

\section{REFERENCES}

1 Trulock EP, Edwards LB, Taylor DO, et al. The registry of the International Society for Heart and Lung Transplantation: twentieth official adult heart-lung report - 2003. J Heart Lung Transplant 2003; 22: 625-635.

2 Reichenspurner H, Girgis RE, Robbins BC, et al. Stanford experience with obliterative bronchiolitis after lung and heart-lung transplantation. Ann Thorac Surg 1996; 62: 1467-1472.

3 Meyers BF, Lynch J, Trulock EP, Guthrie TJ, Cooper JD, Patterson GA. Lung transplantation: a decade of experience. Ann Surg 1999; 230: 362-370.

4 Whitehead B, Rees P, Sorensen K, et al. Incidence of obliterative bronchiolitis after heart-lung transplantation in children. J Heart Lung Transplant 1993; 12: 903-908.

5 Ouwens JP, van der Mark TW, Koëter GH, de Boer WJ, Grevink RG, van der Bij W. Bronchiolar airflow impairment after lung transplantation: an early and common manifestation. J Heart Lung Transplant 2002; 21: 1056-1061.

6 Van Raemdonck D, Verleden G, Vanhaecke J, et al. The current status of lung transplantation. Acta Chir Austriaca 1999; 31: 292-301.

7 Tamm M, Sharples L, Higenbottam T, Steward S, Wallwork J. Bronchiolitis obliterans syndrome (BOS) following heart-lung transplantation. Transpl Int 1996; 9: Suppl. 1, S299-S302.

8 Glanville AR, Baldwin JC, Burke CM, Theodore J, Robin ED. Obliterative bronchiolitis after heart-lung transplantation: apparent arrest by augmented immunosuppression. Ann Int Med 1987; 107: 300-304.

9 Verleden GM. Bronchiolitis obliterans syndrome after lung transplantation: medical treatment. Monaldi Arch Chest Dis 2000; 55: 140-145.

10 Stewart S. The pathology of lung transplantation. Sem Diagnostic Pathol 1992; 9: 210-219.

11 Pomerance A, Madden B, Burke MM, Yacoub MH. Transbronchial biopsy in heart and lung transplantation: clinicopathologic correlations. J Heart Lung Transplant 1995; 14: 761-763.

12 Kramer MR, Stoehr C, Wang JL, et al. The diagnosis of obliterative bronchiolitis after heart-lung and lung transplantation: low yield of transbronchial biopsies. J Heart Lung Transplant 1993; 12: 675-681.

13 Cooper JD, Billingham $\mathrm{M}$, Egan $\mathrm{T}$, et al. A working formulation for the standardisation of nomenclature and for clinical staging of chronic dysfunction in lung allografts. J Heart Lung Transplant 1993; 12: 713-716.

14 Estenne M, Maurer J, Boehler A, et al. Bronchiolitis obliterans syndrome 2001: an update of the diagnostic criteria. J Heart Lung Transplant 2002; 21: 297-310.
15 Patterson GM, Wilson S, Whang JL, et al. Physiologic definitions of obliterative bronchiolitis in heart-lung and double lung transplantation: a comparison of the forced expiratory flow between $25 \%$ and $75 \%$ of the forced vital capacity and forced expiratory volume in one second. J Heart Lung Transplant 1996; 15: 175-181.

16 Reynaud-Gaubert M, Thomas P, Badier M, Cau P, Giudicelli R, Fuentes P. Early detection of airway involvement in obliterative bronchiolitis after lung transplantation. Functional and bronchoalveolar lavage findings. Am J Respir Crit Care Med 2000; 161: 1924-1929.

17 Palmer SM, Miralles AP, Howell DN, Brazer SR, Tapson VF, Davis RD. Gastroesophageal reflux as a reversible cause of allograft dysfunction after lung transplantation. Chest 2000; 118: 1214-1217.

18 Young LR, Hadjiliadis D, Davis D, Palmer SM. Lung transplantation exacerbates gastroesophageal reflux disease. Chest 2003; 124: 1689-1693.

19 Hadjiliadis D, Davis RD, Steele MP, et al. Gastroesophageal reflux disease in lung transplant recipients. Clin Transplant 2003; 17: 363-368.

20 Davis D, Lau CL, Eubanks SS, et al. Improved lung allograft function after fundoplication in patients with gastroesophageal reflux disease undergoing lung transplantation. J Thorac Cardiovasc Surg 2003; 125: 533-542.

21 Maes BD, Vanwalleghem J, Kuypers D, Ghoos Y, Rutgeerts P, Vanrenterghem Y. Differences in gastric motor activity in renal transplant patients treated with FK-506 versus ciclosporine. Transplantation 1999; 68: 1482-1485.

22 Verleden GM, Besse T, Maes B. Successfull conversion from cyclosporine to tacrolimus for gastric motor dysfunction in a lung transplant recipient. Transplantation 2002; 73: 1974-1976.

23 Schachter LM, Dixon J, Pierce RJ, O’Brien P. Severe gastroesophageal reflux is associated with reduced carbon monoxide diffusing capacity. Chest 2003; 123: 1932-1938.

24 Stanbrook MB, Kesten S. Bronchial hyperreactivity after lung transplantation predicts early bronchiolitis obliterans. Am J Respir Crit Care Med 1999; 160: 2034-2039.

25 Hartwig MG, Cantu E, Appel JZ, Woreta H, Palmer SH, Davis RD. Non-alloimmune injury mediated by gastroesophageal reflux precipitates alloimmune injury in lung transplant patients. J Heart Lung Transplant 2004; 23: Suppl. 1, S43.

26 Gerhardt S, McDyer JF, Girgis RE, Conte JV, Orens JB. Maintenance azithromycin therapy for bronchiolitis obliterans syndrome. Am J Respir Crit Care med 2003; 168: 121-125.

27 Verleden GM, Dupont LJ. Azithromycin therapy for patients with bronchiolitis obliterans syndrome after lung transplantation. Transplantation 2004; 77: 1465-1467.

28 Estenne M, Hertz MI. Bronchiolitis obliterans after human lung transplantation. Am J Respir Crit Care Med 2002; 166: 440-444.

29 Ward C, Camara M, Forrest I, et al. Preliminary findings of quorum signal molecules in clinically stable lung allograft recipients. Thorax 2003; 58: 444-446.

30 DiGiovine B, Lynch JP 3rd, Martinez FJ, et al. Bronchoalveolar lavage neutrophilia is associated with obliterative bronchiolitis after lung transplantation. Immunol 1996; 157: 4194-4202. 